\title{
Marketing de intangíveis: A servicescape e o uso das evidências físicas para a projeção dos ambientes de serviço
}

\author{
Intangible Marketing: The servicescape and the use of physical evidence projection of service environments
}

\section{Marcelo da Silva Schuster}

Universidade Federal de Santa Maria, Departamento de Administração, Curso de Doutorado em Administração, Santa Maria, RS, Brasil, 97105-900 marcelo.schuster@gmail.com

\section{Valéria da Veiga Dias}

Universidade Federal do Rio Grande do Sul, Centro de Estudos em Agronegócio, Curso de Doutorado em Agronegócios, Porto Alegre, RS, Brasil,91540000 valeria-adm@hotmail.com

\section{Luciana Flores Battistella}

Universidade Federal de Santa Maria, Departamento de Administração, Curso de Doutorado em Administração, Santa Maria, RS, Brasil, 97105-900 lutti@ufsm.br

\section{Resumo}

Considerando as inúmeras influências nas percepções do consumidor, existentes no ambiente de serviço, este estudo objetivou criar um framework sobre uso da servicescape. Para isso, foram analisadas escalas, variáveis, dimensões, ambientes e construtos utilizados em publicações anteriores. A metodologia escolhida foi a bibliometria, com uso da base de dados Scopus. Dos 85 artigos selecionados, 36 utilizaram algum tipo de escala de servicescape. Estes foram analisados de forma manual e individual por meio da análise de conteúdo. A maior parte dos estudos realizou pesquisas com consumidores e o ambiente preferido foi o de hospitalidade (shoppings, restaurantes, hotéis). O framework resultante representa uma diversidade de variáveis, fatores e construtos, inerentes a uma discussão da influência do ambiente de serviços para os estudos do consumidor. Este estudo contribui para o estado da arte sobre o tema, mas principalmente para embasar a construção de uma métrica adequada e futuros estudos empíricos.

Palavras-chave: Servicescape, framework, marketing de serviços.

\section{Abstract}

Considering the numerous influences in consumer perceptions, existing in the service environment, this study aimed to create a framework on use of servicescape. Has been analyzed scales used, variables, dimensions, environments and constructs used in previous publications. The chosen methodology was bibliometrics, using the Scopus database. Of the 85 selected articles, 36 used some kind of servicescape scale. These were analyzed manually and individually by content analysis. Most studies conducted research with consumers and the most used environment was hospitality (malls, restaurants, hotels). The resulting framework reflects the plurality of variables, factors and constructs linked to a discussion about service environment on consumer studies. This study contributes literature on the subject, but mainly for the construction of appropriate metric and future empirical studies.

Keywords: Servicescape; framework; service marketing

\section{Introdução}

O consumidor é amplamente influenciado, em suas decisões, por uma grande diversidade de elementos no ambiente de consumo. Este consumidor tende a avaliar os locais frequentados de forma holística, e essa avaliação muitas vezes depende das impressões ou percepções a respeito dos elementos do ambientes físico (Lin, 2004)

A importância destes elementos do ambiente é destacada no marketing, principalmente no marketing de serviços, que se baseia nas experiências vivenciadas pelos consumidores e como estas influenciam suas decisões no futuro. Solomon, Marshall e Stuart (2015) destacam que as experiências desejadas pelos consumidores são induzidas "de" ou "sobre" alguma coisa, por meio de estímulos ambientais. Além disso, Lam, Chan, Fong e Lo (2011) afirmam que as experiências de consumo são influenciadas pelas emoções dos consumidores, geradas a partir do contato com determinado ambiente.

Tais influências se originam das características particulares dos serviços, como a intangibilidade, a inseparabilidade e a perecibilidade que exigem que o consumidor encontre mecanismos para a avaliação do serviço oferecido (Solomon, Marshall, \& Stuart, 2015). De acordo com estes autores um dos principais meios de avaliação do serviço prestado é a avaliação do local onde o serviço é oferecido.
Inicialmente a avaliação dos serviços incluiu apenas o ambiente físico da loja (Kotler, 1973), os elementos nomeados como fatores atmosféricos. De acordo com Bitner (1992) a avaliação dos serviços deve ser realizada a partir da análise da "servicescape" definida como o conjunto de estímulos físicos e sociais existentes no ambiente. Este conceito, inicialmente foi estruturado a partir de três dimensões: (a) Condições Ambientais - temperatura, qualidade do ar, barulho, música cheiro, etc; (b) Espaço e funcionalidades - layout do local, equipamentos utilizados, mobília, e outros; e (c) Sinais, Símbolos e Artefatos - estilo e decoração do ambiente, artefatos pessoais e sinalizações do local (Bitner,1992). Posteriormente, a dimensão socialfoi incluída como elemento relevante na análise do ambiente (Rosenbaum \& Montoya, 2007). Desta forma, a avaliação do serviço está relacionada aos fatores sociais, onde se estabelece a relação entre colaboradores e clientes que frequentam o local; e o ambiente físico (interno externo), onde ocorre a interação entre a organização, o consumidor e o serviço entregue (Solomon, Marshall \& Stuart, 2015).

Ao longo dos anos, diversos estudos procuraram mensurar os diversos elementos do ambiente de serviço a partir da servicescape, no entanto, ainda não há consenso na literatura sobre os elementos essenciais para a investigação do ambiente. Esta indefinição entre as escalas e dimensões motivou este estudo, 
visto que, a importância das características de avaliação dos serviços cresce na medida em que os resultados financeiros atrelados ao setor de serviços ganham destaque na economia e na agregação de valor de grande parte das organizações. Em 2013, o setor de serviços foi responsável por quatro em cada cinco postos de trabalho nos estados Unidos e quase 80 por cento do Produto Interno Bruto (PIB) (Solomon, Marshall, \& Stuart, 2015) e no Brasil $70,8 \%$ do PIB é correspondente a serviços (IBGE, 2015) e destes 9,3\% ou 492 bilhões de reais estão atrelados a serviços de hospitalidade (Braga, 2015).

Diante do papel de destaque dos serviços na atualidade, bem como da dificuldade de compreender as influências do ambiente nas ações do consumidor, cabe o questionamento sobre como capturar as percepções, impressões, sensações e emoções de um consumidor a respeito do local de compra ou consumo? Considerando o esforço já realizado por estudiosos na tentativa de organizar a servicescape e suas dimensões, este estudo se propôs a investigar, na literatura nacional e internacional, a aplicação desta e sua interação com comportamentos do consumidor. A intenção dos pesquisadores foi de apresentar um framework referente ao tema a partir das variáveis e dimensões utilizadas em estudos anteriores.

\section{Servicescape}

O marketing de serviços diferencia-se do marketing de produtos, principalmente em função da sua intangibilidade. Em comparação aos bens, os serviços entregam um pacote de benefícios por meio da experiência criada para o consumidor. $O$ desafio, que em geral não existe para os bens tangíveis. Tais desafios incluem a impossibilidade de estoque, a falta de proteção de patentes, dificuldade de padronização, perecibilidade, dificuldade de comunicação dos atributos ao mercado-alvo, entre outros (Bateson \& Hoffman, 2016). Desta forma, há necessidade de adequação estratégia e do mix de marketing tradicional (produto, preço, promoção e praça), incluindo a influência das pessoas, dos processos e das evidências físicas para atender e comunicar-se com os clientes (Zeithaml, Bitner \& Gremler, 2014).

A partir destas características, o estudo dos fatores que influenciam a percepção de qualidade e satisfação do consumidor foi representado por uma série de modelos, aprimorados ao longo da evolução dos estudos em marketing de serviços. Entre estes, estão modelos que mensuram a Qualidade dos Serviços (SERVQUAL) de Parasuraman, Zeithaml, \& Berry (1985), Desempenho do Serviço (SERVPERF) de Cronin e Taylor (1992), Qualidade Interna (INTQUAL) de Caruana e Pitt (1997). Estes modelos e escalas multidimensionais buscam capturar as percepções, e expectativas dos consumidores que acabam por influenciar o relacionamento entre consumidor e empresas de serviço (Zeithaml, Bitner, \& Gremler, 2014).

Devido a intangibilidade dos fatores associados ao serviço, os consumidores terão dificuldade de avaliar objetivamente a qualidade dos serviços. Muitas vezes estes consumidores usam evidências tangíveis em torno do serviço para formar avaliações. O papel das evidências físicas no marketing de intangíveis possui muitas facetas e influências na diferenciação do serviço diante do consumidor. Desta forma, ao passo que aumenta a relevância dos serviços na economia das nações e organizações, bem como o conhecimento relacionado ao seu uso como vantagem competitiva, a compreensão do que se nomeou como servicescape vem ganhando destaque.

A servicescape pode ser conceituada como o uso das evidências físicas para projetar ambientes de serviço (Bateson \& Hoffman, 2016). Dentre os estudos relacionados ao tema, é possível indicar que as primeiras pesquisas partiram da identificação da importância do papel da imagem do local e seus múltiplos elementos na avaliação do consumidor.

A investigação da importância da imagem, como um dos fatores relevantes na influência da percepção sobre o ambiente de serviços, foi pesquisada desde a década de 1950. Neste período Martineau (1958) caracterizou e discutiu a questão do poder da imagem do local sobre os consumidores, estabelecendo os fatores de maior relevância para a avaliação dos mesmos, tais como layout e arquitetura; símbolos e cores; publicidade e os vendedores.

Em consonância com os estudos dos ambientes de serviços Kotler (1973) nomeou como fatores atmosféricos, os elementos responsáveis por "projetar o ambiente de compras para produzir efeitos emocionais específicos no comprador que aumentem sua probabilidade de compra" (Kotler, 1973, p 50-51). Mari e Poggesi (2013) explicam que a atmosfera de um ambiente é descrita em termos sensoriais, onde os principais canais sensoriais para atmosfera são visão, som, cheiro e toque, podendo afetar o comportamento de compra, por meio do despertar de reações viscerais que podem contribuir favoravelmente para a probabilidade de comprar.

Posteriormente, em 1992, Mary Jo Bitner compilou estudos anteriores e cunhou o termo servicescape para descrever como prestadores de serviços usam os fatores físicos do ambiente para influenciar o comportamento dos consumidores (Lee, Wang, \& Cai, 2015). Esses fatores foram estruturados em três categorias: Condições Ambientais; Espaço e Funcionalidades; e Sinais, Símbolos e Artefatos. Resgatando os estudos de Martineau (1958), em 2007 Mark Rosenbaum e Detra Montoya (Rosenbaum \& Montoya, 2007), incluiram o fator social aos estudos da servicescape, incluindo clientes e colaboradores como elementos do ambiente de consumo. Os fatores da servicescape foram apresentados na Tabela 1.

\section{Tabela 1 - Fatores da Servicescape}

\begin{tabular}{l|l}
\multicolumn{1}{c|}{ Fator } & \multicolumn{1}{c}{ Conceito } \\
\hline $\begin{array}{l}\text { Condições } \\
\text { Ambientais (CA) }\end{array}$ & $\begin{array}{l}\text { Incluem características de fundo do ambiente, } \\
\text { como temperatura, iluminação, ruído, música e } \\
\text { perfume }\end{array}$ \\
\hline $\begin{array}{l}\text { Refere-se às formas em que máquinas, } \\
\text { equipamentos e mobiliário são organizados, o } \\
\text { tamanho e a forma de esses itens, e as relações } \\
\text { Funcionalidades } \\
\text { (FU) }\end{array}$ & $\begin{array}{l}\text { espaciais entre eles. 'Funcionalidade' refere-se à } \\
\text { capacidade de os mesmos itens para facilitar o } \\
\text { desempenho e a realização de objetivos }\end{array}$ \\
\hline $\begin{array}{l}\text { Sinais, Símbolos } \\
\text { e Artefatos (SI) }\end{array}$ & $\begin{array}{l}\text { São ferramenta para transmitir sinais tanto } \\
\text { implícitos e explícitos e comunicar informações } \\
\text { sobre um lugar para usuários ou clientes }\end{array}$ \\
\hline Social (SO) & $\begin{array}{l}\text { Definida como contendo estímulos dos } \\
\text { funcionários, clientes, densidade social } \\
\text { (aglomeração) e exibindo emoções dos outros } \\
\text { sobre os consumidores }\end{array}$
\end{tabular}

Fonte: Adaptado de diversos autores (Lam et al., 2011; Rosenbaum \& Massiah, 2011; Lee, Wang \& Cai, 2015). 
Mesmo com a aparente evolução dos estudos da servicescape, não existe um consenso sobre a quantidade de fatores da escala, bem como que variáveis devem ser pesquisadas, alteradas de autor para autor. Para diversos pesquisadores a escala é tri dimensional (três fatores) conforme proposto por Bitner (Hightower Jr, Brady, $\&$ Baker, 2002), outros pesquisadores optam por pesquisar apenas um ou dois desses fatores (Reynolds \& Harris, 2009; Wang, Hsu, \& Fang, 2009; Daunt \& Harris, 2012a) e há aqueles que utilizam escalas de mensuração geral, sem identificar nenhum fator ou dimensão específica para avaliar a servicescape (Grace \& O'Cass, 2005; Chiou \& Chen, 2012; Chang, Jeng, \& Hamid, 2013).

Tais diferenças e a falta de uma escala consolidada para avaliar a servicescape (ambiente de serviço) motivaram a elaboração deste artigo, que buscou avaliar todos os estudos já publicados, que utilizaram escalas para mensurar a servicescape. Investigando ainda em quais ambientes os estudos foram aplicados, quais fatores ou dimensões foram utilizados, o público alvo, os construtos que se relacionaram e as variáveis utilizadas para mensurar a servicescape. A seção seguinte detalha melhor o método utilizado nesse estudo.

\section{Metodologia}

Com o intuito de obter um levantamento representativo sobre os estudos e a mensuração do ambiente de serviço (Servicescape), optou-se pela consulta de uma base de dados. A ISI Web Of Science (WoS) foi por muitos anos a principal base de dados para publicações do mundo todo, pois era a única que permitia compilar dados e contribuir para estudos bibliométricos. No entanto, em 2004 a editora Elsevier lançou a base Sci-Verse- Scopus e segundo a própria editora, além da concorrência com a WoS, se constituiu no maior banco de dados multidisciplinar do mundoi. São mais de 46 milhões de registros e aproximadamente 19.500 títulos provenientes de 5.000 editoras do mundo possibilitando ao pesquisador obter informações suficientes para basear seus projetos, desde a pesquisa básica, aplicada e até mesmo a inovação tecnológica. Optou-se, portanto pela pesquisa utilizando como fonte de pesquisa a base Scopus (SCI VERSE, 2015).

A escolha da pesquisa da escala servicescape se deu em função dos reflexos do setor de serviços na economia de diversos países, inclusive o Brasil, e em função da necessidade de compreensão das dimensões da escala na estrutura das organizações. Por fim observa-se na literatura que as formas de mensuração desta ainda estão em fase de estruturação e consolidação, permitindo abordagens diferentes em estudos organizacionais da área de marketing/comportamento do consumidor. Diante disto, este estudo buscou compilar em um framework, de que forma essa mensuração é utilizada nos estudos sobre o tema.

Para operacionalização da pesquisa foi utilizada a "proxy" da Universidade Federal de Santa Maria, ampliando o acesso às publicações da base de dados SCOPUS. A busca utilizou a palavrachave "Servicescape" e a busca foi realizada considerando Article, Titles e Abstract, onde foram encontrados 189 trabalhos relacionados com a temática. Em seguida, foi realizado um refinamento e uma nova busca, usando a palavra "scale", com intenção de restringir a busca aos estudos que utilizaram algum tipo de escala referente a mensuração da servicescape. Dos 189 estudos identificados, restaram 89 artigos, dos quais foi possível acessar 85 .

Para a análise dos artigos foi escolhida e técnica de análise de conteúdo, conforme sugerida por Bardin (2010), que implica em quatro etapas: i) Leitura flutuante do material a ser analisado, neste caso os 89 trabalhos identificados na pesquisa da base de dados; ii) Identificação e seleção do conteúdo de análise; ou seja, organização do material a ser considerado; iii) Categorização (baseado no tema, contagens e outros parâmetro previamente estabelecidos), neste caso, inicialmente os artigos foram organizados a partir do tipo de ambiente de serviço encontrado nos artigos, construtos pesquisados, estrutura das escalas e variáveis utilizadas para mensurar a servicescape ; iv) Análise comparativa ou interpretativa do conteúdo. Esta análise foi realizada manualmente e de forma individual. As estratégias de seleção e exclusão dos estudos foram descritas conforme descrito na Tabela 2.

Tabela 2 - Critérios de exclusão de artigos da análise

\begin{tabular}{|l|l|}
\hline \multicolumn{1}{|c|}{ Requisitos para exclusão } \\
\hline $\mathbf{1}$ & $\begin{array}{l}\text { Para ser selecionado para análise o artigo deve ter utilizado } \\
\text { alguma escala para mensurar a servicescape. Além disso, o } \\
\text { estudo deve apresentar a composição desta escala. Caso } \\
\text { estes critérios não tenham sido atendidos o artigo será } \\
\text { excluído da amostra dos } 89 \text { trabalhos; }\end{array}$ \\
\hline $\mathbf{2}$ & Artigos exclusivamente teóricos foram excluídos da análise; \\
\hline $\mathbf{3}$ & $\begin{array}{l}\text { Trabalhos que apenas mencionaram escalas de outros } \\
\text { autores e não apresentaram sua estrutura foram } \\
\text { investigadas, ou seja, foi realizada uma busca na base citada } \\
\text { e caso a escala não tenha sido encontrada, o artigo foi } \\
\text { excluído da análise; }\end{array}$ \\
\hline $\mathbf{4}$ & $\begin{array}{l}\text { Artigos que pesquisaram apenas uma variável da } \\
\text { servicescape (ex: música aroma, etc.), sem utilizar uma } \\
\text { escala, foram excluídos. }\end{array}$ \\
\hline
\end{tabular}

Foram excluídos quatro artigos que não apresentarem a escala utilizada de forma explicita e outros quatro que utilizaram adaptações de escalas, com menor número de questões do que a escala original, impossibilitando a verificação das variáveis utilizadas. Em função destes critérios de seleção restaram 42 artigos, que foram analisados.

Para aumentar a validade deste estudo optou-se por estruturar um mecanismo de verificação dos resultados. O primeiro pesquisador classificou os resultados a partir dos parâmetros estabelecidos (ambiente de serviço, construtos pesquisados, estrutura das escalas e variáveis utilizadas para mensurar a servicescape), o segundo pesquisador faz a mesma classificação, nos casos de divergência o estudo foi encaminhado para um terceiro pesquisador que avaliou a coerência da divergência e a classificação mais adequada.

\section{Resultados}

Nos últimos anos, diversos estudos têm procurado identificar e explorar a relação entre fatores da servicescape e resultados comportamentais dos usuários finais (Lee $\&$ Kim, 2014). A análise dos resultados segue a sequência de público-alvo, Ambiente, Construtos, Fatores e Variáveis. Analisando o público alvo dos artigos selecionados, foi constatado que somente dois estudos (Naqshbandi, 
Muzamil, \& Munir, 2011; Koo, Andrew, \& Kim, 2008) não utilizaram consumidores para avaliar a servicescape e sim estudantes.

Diversos ambientes serviram como plano de fundo para os estudos investigados, estes foram apresentados na Tabela 3 a seguir, com a descrição do local, autores e ano da pesquisa. Destaca-se que o ambiente mais pesquisado é aquele chamado de ambiente de serviços de hospitalidade, descrito por (Morrison, 2012) como ambientes que envolvem o entretenimento, restaurantes e hotéis, que fornecem serviços aos clientes proporcionando experiências de entretenimento.

Tabela 3 - Ambientes investigados nos estudos publicados na base de dados Scopus a respeito do tema servicescape

\begin{tabular}{|c|c|}
\hline Tipo de ambiente de serviço & Autor/ano \\
\hline $\begin{array}{l}\text { Atendimento/Hospitalidade: } \\
\text { (shopping, parques, restaurantes, } \\
\text { hotéis, centros desportivos) }\end{array}$ & $\begin{array}{l}\text { Leblanc e Nguyen (1996); Hightower Jr, Brady e Baker (2002); Grace e O'cass (2005); Reimer e } \\
\text { Kuehn (2005); Nguyen (2006); Harris e Ezeh (2008); Koo, Andrew e Kim (2008); O'cass e Grace } \\
\text { (2008); Kim e Moon (2009); Reynolds e Harris (2009); Riviezzo, De Nisco e Napolitano (2009); } \\
\text { Wang, Hsu e Fang (2009); Bruggen, Foubert e Gremler (2011); Ismail (2011); Minkiewicz, Evans, } \\
\text { Bridson, e Mavondo (2011); Naqshbandi, Muzamil e Munir (2011); Daunt e Harris (2012a; b); } \\
\text { Miles, Miles e Cannon (2012); Nguyen, Dewitt e Russell-Bennett (2012); De Nisco e Warnaby } \\
\text { (2013); Dong e siu (2013); Hightower (2013); Campbell e Dipietro (2014); Fernandes e Neves } \\
\text { (2014); Lim (2014); Lund e Marinova (2014); Chen, Raab e Tanford (2015); Collier e Barnes } \\
\text { (2015); Lee, Wang e Cai (2015). }\end{array}$ \\
\hline Educacionais & Chang, Jeng e Hamid (2013) \\
\hline Transportes & Pantouvakis (2010); Chiou e Chen (2012); Hooper, Coughlan e Mullen (2013) \\
\hline Online & Tuzovic (2008); Harris e Goode (2010); Roy, Lassar e Butaney (2014) \\
\hline
\end{tabular}

A avaliação da servicescape parece abranger diversas áreas, porém as referências mais encontradas são para serviços de hospitalidade (shoppings, hotéis, restaurantes, etc). corroborando com (Lin, 2004) que afirma que a servicescape é um importante componente tangível do serviço criando uma imagem perceptual de imediato na mente dos clientes através das pistas fornecidas por este ambiente.

A importância da servicescape sobre as intenções comportamentais dos consumidores já foi discutida por diversos autores (Kotler, 1973; Bitner, 1992; Zeithaml, Bitner, \& Gremler, 2014) que reverberam a influencia no comportamento de compra dos clientes e na criação de uma imagem particularmente evidente para os serviços. Dentro desta proposta temática (Ellen \& Zhang, 2014) usando equações estruturais constataram que a servicescape influencia os estados emocionais (prazer e excitação) dos consumidores e através destas emoções, as suas intenções comportamentais. Já Lam et al. (2011) confirmou a influência na vontade do consumidor em permanecer no local.

Diversos construtos aparecem relacionados com a aplicação da servicescape, neste estudo foram encontrados oito construtos mais pesquisados, conforme a Tabela 4 a seguir:

Tabela 4 - Construtos identificados nos estudos publicados na base de dados Scopus a respeito do tema servicescape

\begin{tabular}{|l|l|}
\hline \multicolumn{1}{|c|}{ Construto } & \multicolumn{1}{c}{ Autor/ano } \\
\hline Satisfação & Koo, Andrew e Kim (2008); Miles, Miles e Cannon (2012); Hightower (2013); Fernandes e Neves (2014) \\
\hline $\begin{array}{l}\text { Qualidade do Serviço } \\
\text { Confiança }\end{array}$ & Chiou e Chen (2012); Hooper, Coughlan e Mullen (2013); Chang, Jeng e Hamid (2013) \\
\hline Lealdade & Reimer e Kuehn (2005); Wang, Hsu e Fang, (2009); Harris e Goode (2010) \\
\hline Recompra & Kim e Moon (2009); Campbell e Dipietro (2014) \\
\hline Imagem & Leblanc e Nguyen (1996); Nguyen (2006); Minkiewicz et al. (2011) \\
\hline Descontentamento do cliente & Daunt e Harris (2012a) \\
\hline Passa a Palavra (Word of Mouth) & Bruggen, Foubert e Gremler (2011); Roy, Lassar e Butaney (2014) \\
\hline
\end{tabular}

Além destes, outros construtos aparecem de forma menos destacada. Evidencia-se por meio desses construtos a importância da servicescape para as organizações, já que estes se relacionam com a longevidade das organizações. Esta longevidade esta associada principalmente a satisfação dos consumidores e sua conseqüente lealdade, a insatisfação e a falta de lealdade diminuem também a chance de que o consumidor retorne ao local de compra e reverbere suas experiências aos amigos.

Quanto às escalas, foi encontrada muita discordância no que tange a sua construção e estruturação. Foram encontradas escalas que utilizaram três variáveis para mensurar a servicescape (Chiou \& Chen, 2012; Nguyen, Dewitt, \& Russell-Bennett, 2012; Pantouvakis, 2012) até escalas que utilizam 49 (Harris \& Goode, 2010), sendo assim para melhor compreensão da estrutura e análise das escalas foi utilizado o critério da quantidade de fatores ou dimensões proposto por Bitner (1992). A partir da proposta tretradimensional realizou-se uma comparação e adequação da nomenclatura de cada autor conforme essa proposta. Para melhor compreensão apresentou-se a Tabela 5 a seguir: 
Tabela 5 - Tipos e estrutura das escalas encontradas para a mensuração da servicescape

\begin{tabular}{|c|c|c|c|}
\hline Tipo escala & $\begin{array}{l}\text { Quantidade } \\
\text { de estudos }\end{array}$ & Dimensões ou Fatores & Autor/ano \\
\hline Geral $^{1}$ & 16 & Nenhum & $\begin{array}{l}\text { Hightower Jr, Brady e Baker (2002); Grace e O'cass (2005); Reimer e } \\
\text { Kuehn (2005); Nguyen (2006); O'cass e Grace (2008); Pantouvakis } \\
\text { (2010); Bruggen, Foubert e Gremler (2011); Ismail (2011); Chiou e } \\
\text { Chen (2012); Pantouvakis (2012); Chang, Jeng e Hamid (2013); } \\
\text { Hightower (2013); Campbell e Dipietro (2014); Fernandes e Neves } \\
\text { (2014); Lim (2014); Collier e Barnes (2015). }\end{array}$ \\
\hline Tetradimensional & Nenhum & Nenhum & Nenhum \\
\hline \multirow{3}{*}{ Tridimensional } & \multirow{3}{*}{ Oito (8) } & $\begin{array}{l}\text { Tabela (4) Condições Ambientais, } \\
\text { Espaço e Funcionalidades, e Sinais, } \\
\text { símbolos e artefatos }\end{array}$ & $\begin{array}{l}\text { Kim e Moon (2009); Minkiewicz et al. (2011); Miles, Miles e Cannon } \\
\text { (2012); Roy, Lassar \& Butaney (2014) }\end{array}$ \\
\hline & & $\begin{array}{l}\text { Três (3) Condições Ambientais, } \\
\text { Espaço e Funcionalidades, e Social }\end{array}$ & $\begin{array}{l}\text { Harris e Ezeh (2008); Reynolds e Harris (2009); Riviezzo, De Nisco e } \\
\text { Napolitano (2009) }\end{array}$ \\
\hline & & $\begin{array}{l}\text { Um (1) Condições Ambientais, } \\
\text { Sinais, símbolos e artefatos, e Social }\end{array}$ & Dong e Siu (2013). \\
\hline \multirow{2}{*}{ Bidimensional } & \multirow{2}{*}{ Dez (10) } & $\begin{array}{l}\text { Oito (8) Condições Ambientais, } \\
\text { Espaço e Funcionalidades }\end{array}$ & $\begin{array}{l}\text { Leblanc e Nguyen (1996); Koo, Andrew e Kim (2008); Wang, Hsu e } \\
\text { Fang (2009); Naqshbandi Muzamil e Munir (2011); Daunt e Harris } \\
\text { (2012b); Hooper, Coughlan e Mullen (2013) }\end{array}$ \\
\hline & & $\begin{array}{l}\text { Dois (2) Espaço e Funcionalidades, e } \\
\text { Sinais, símbolos e artefatos }\end{array}$ & Newman (2007); Harris e Goode (2010) \\
\hline \multirow[t]{2}{*}{ Unidimensional } & \multirow[t]{2}{*}{ Dois (2) } & Um (1) Sinais, símbolos e artefatos & Daunt e Harris (2012a) \\
\hline & & Um (1) Social & Nguyen, Dewitt e Russell-Bennett (2012). \\
\hline
\end{tabular}

${ }^{1}$ A diferença entre essa estrutura e a unidimensional é que na escala geral são misturados vários dos quatro fatores (dimensões), enquanto na escala unidimensional os autores escolhem uma das quatro dimensões.

No que tange às variáveis pesquisadas, estas diferem muito entre os estudos, por isso foram agrupadas semanticamente, ou seja, pelo sentido da pergunta. A Tabela 6 apresenta as variáveis encontradas, assim como a frequência que elas apareceram e a quantidade de perguntas associadas a elas.

Tabela 6 - Variáveis mais encontradas nos estudos da servicescape

\begin{tabular}{|c|c|c|c|}
\hline Variável & Frequência & Perguntas & Fator \\
\hline Aglomeração & 6 & 6 & so \\
\hline Aparência Física & 2 & 2 & $\mathrm{SI}$ \\
\hline Arquitetura e Design & 29 & 28 & $\mathrm{SI}$ \\
\hline Barulho & 4 & 3 & CA \\
\hline Cheiro & 11 & 10 & CA \\
\hline Competência do Funcionário & 5 & 3 & so \\
\hline Comportamento outros clientes & 6 & 6 & so \\
\hline Conforto & 5 & 5 & FU \\
\hline Cores & 13 & 12 & CA \\
\hline Disposição Espacial & 22 & 22 & FU \\
\hline Entretenimento & 2 & 2 & FU \\
\hline Equipamentos de Mídia & 3 & 3 & FU \\
\hline Estacionamento & 3 & 3 & FU \\
\hline Aparência dos Funcionários & 10 & 9 & so \\
\hline Interatividade & 4 & 4 & so \\
\hline Limpeza & 17 & 16 & CA \\
\hline Localização - área & 4 & 4 & FU \\
\hline Luz & 14 & 12 & CA \\
\hline Mobília & 5 & 5 & FU \\
\hline Musica & 10 & 9 & CA \\
\hline Qualidade do Ar & 1 & 1 & CA \\
\hline Segurança & 9 & 4 & CA \\
\hline Sentimento de pertença & 4 & 4 & so \\
\hline Sinalização & 14 & 13 & $\mathrm{SI}$ \\
\hline Temperatura & 10 & 9 & CA \\
\hline
\end{tabular}

Cabe ressaltar que a estruturação de cada variável assim como a nomeação dos fatores é volátil, uma vez que cada autor realizou sua classificação particular. Desta forma, elaborou-se uma classificação abrangente, visando colaborar com novos estudos sobre o tema. A Figura 1 representa o esforço de reunir os achados de pesquisa sobre mensurações de servicescape na forma de um framework.
O framework apresentado reuniu as variáveis associadas a cada um dos quatro fatores/dimensões, procurando clarificar quais seriam as variáveis formadoras destes. Além disso, foram analisados os construtos estudados em conjunto com a servicescape, ilustrando que preocupações permeiam as pesquisas associadas ao marketing de serviço e a compreensão dos ambientes de serviço. 
Figura 1 - Framework referente às dimensões, fatores e variáveis estruturados em formas quantitativas de mensuração da Servicescape

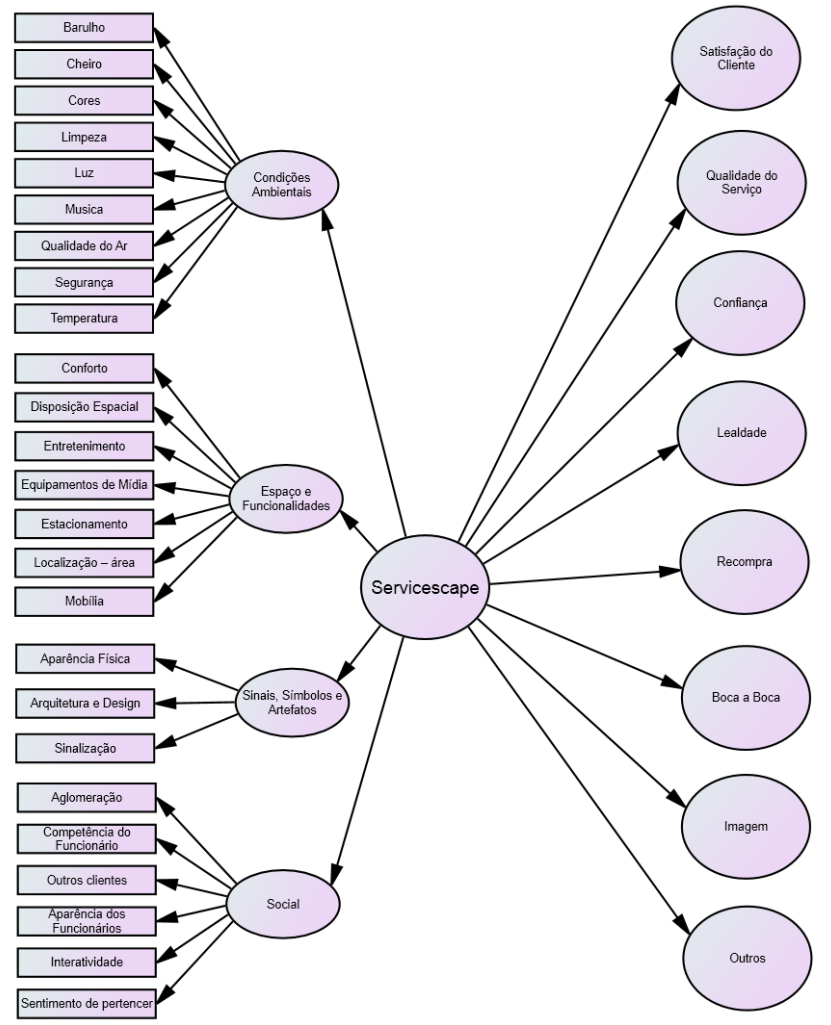

Os achados deste estudo não têm intenção de esgotar o tema, mas ilustrar, por meio desta representação de que forma os estudos já realizados abordaram a questão e que fatores e variáveis foram mais utilizados para mensurar a servicescape. Desta forma, trazendo reflexões sobre o tema e sua relevância para os estudos do consumidor.

\section{Considerações Finais}

A servicescape é uma importante fonte de evidência na formação de impressões de um consumidor sobre um ambiente de serviços. A avaliação dos aspectos ambientais, como a arquitetura, design, disposição dos colaboradores e até mesmo a interação com outros clientes que frequentam o local, ajuda a moldar a imagem da organização, dos produtos e dos serviços perante o consumidor.

Este tema começou a ser estudado na década de 1950 e até hoje encontra-se em estruturação, tendo este estudo objetivado analisar os ambientes que foram pesquisados, fatores ou dimensões, o público alvo dos estudos, os construtos que se relacionaram e as variáveis utilizadas para mensurar a servicescape. Observou-se que o ambiente mais pesquisado foi o de Serviços de Hospitalidade, que inclui Shopping, parques, restaurantes, hotéis, centros desportivos, com destaque para as datas de publicação que em geral são de 2010 a 2015. Construtos relevantes para a área de negócios e gestão, bem como para o delineamento estratégico das organizações se destacaram nas pesquisas envolvendo o ambiente de serviço e a servicescape, com destaque para satisfação, confiança, qualidade percebida, lealdade, recompra, etc. Diversos destes, vinculados com pesquisas sobre a lealdade dos clientes, continuidade e marketing de relacionamento, inovação de produtos entre outros.

Considerou-se que o framework apresentado representa a importância do tema para as organizações, além de estabelecer a necessidade de novos estudos e da criação de uma escala abrangente com os fatores encontrados. É visto que a utilização da escala difere conforme os objetivos de pesquisa dos autores, assim como os construtos relacionados, porém na análise dos estudos foi verificado que muitas escalas são estruturadas por meio de adaptações de diversas outras, sendo que poucas abordam a validação dessas. No entanto, a estrutura mais utilizada foi a que não define dimensões ou fatores (16 estudos), mas mescla elementos diversos dos quatro fatores ou dimensões (condições ambientais, espaço e funcionalidades, sinais e social) para avaliar o ambiente. Na sequencia, a estrutura com três dimensões foi a mais aplicada (8 estudos).

A evidência de que a maioria dos estudos utiliza uma escala "geral" (sem delimitação de dimensões) sugere que podem haver lacunas de pesquisa prementes que indicam a necessidade de mais estudos empíricos para comprovar ou diferenciar instrumentos de mensuração para a servicescape.

Apesar da relevância comprovada para o marketing e para as organizações em geral, ainda há um amplo campo de desenvolvimento acadêmico, sendo necessários estudos empíricos futuros aplicando uma forma de mensurar a servicescape. Faltam estudos nacionais, bem como uma escala adequada a realidade brasileira. Estudos posteriores podem ainda ser realizados para elaborar modelos e relações estruturais na área de comportamento do consumidor e marketing de serviço. Como limitações, este estudo utilizou uma única base de dados para pesquisa, que apesar de sua relevância pode constituir-se como um filtro. Conforme indicado nas sugestões, pode-se mencionar como limitação o fato de não terem sido encontradas publicações nacionais. 


\section{Referências}

Altschwager, T., Habel, C., \& Goodman, S. P. (2011). The servicescape response: do brand committed consumers respond differently to the cellar door experience? In: International Conference of the Academy of Wine Business Research 6th Bordeaux, France.

Bardin, L.(2010). Análise de conteúdo. Lisboa: Edição 70.

Bitner, M. J. (1992) Servicescapes: the impact of physical surroundings on customers and employees. The Journal of Marketing, 56 (2), 57-71.

Braga, G. H. (2015). Turismo movimenta R\$ 492 bilhões no Brasil. Recuperado em 01 de fevereiro de 2016, de http://www.turismo.gov.br/ultimas-noticias/957-turismomovimenta-r-492-bilhoes-no-brasil.html.

Bruggen, E. C., Foubert, B., \& Gremler, D. D. (2011). Extreme Makeover: Short- and Long-Term Effects of a Remodeled Servicescape. Journal of Marketing, 75 (5) 71-87.

Campbell, J. M., \& DiPietro, R. B. (2014). Sign of the times: Testing consumer response to local food signage within a casual dining restaurant. Journal of Retailing and Consumer Services, 21 (5) 812-823.

Caruana, A., \& Pitt, L. (1997). INTQUAL-an internal measure of service quality and the link between service quality and business performance. European Journal of Marketing, 31(8), 604-616.

Chang, H. H., Jeng, D. J. F., \& Hamid, M. R. A. (2013) Conceptualising consumers' wordof-mouth behaviour intention: Evidence from a university education services in Malaysia. Service Business. 7 (1) 17-35.

Chen, S. C., Raab, C., Tanford, S. (2015). Antecedents of mandatory customer participation in service encounters: An empirical study. International Journal of Hospitality Management. 46. 65-75.

Chiou, Y. C., \& Chen, Y. H. (2012). Service quality effects on air passenger intentions: A service chain perspective. Transportmetrica. 8 (6) 406-426.

Collier, J. E., Barnes, D. C. (2015). Self-service delight: Exploring the hedonic aspects of self-service. Journal of Business Research. 68 (5) 986-993.

Cronin Jr, J. J., \& Taylor, S. A. (1992). Measuring service quality: a reexamination and extension. The journal of marketing. 56 (3) 55-68.

Daunt, K. L., \& Harris, L. C. (2012a) Exploring the forms of dysfunctional custome behaviour: A study of differences in servicescape and customer disaffection with service. Journal of Marketing Management. 28 (1-2) 129-153.

Daunt, K. L., \& Harris, L. C. (2012b). Motives of dysfunctional customer behavior: An empirical study. Journal of Services Marketing. 26 (4) 293-308.

De Nisco, A., \& Warnaby, G. (2013). Shopping in downtown: The effect of urban environment on service quality perception and behavioural intentions. International Journal of Retail and Distribution Management. 41 (9) 654-670.

Dong, P., \& Siu, N. Y. M. (2013). Servicescape elements, customer predispositions and service experience: The case of theme park visitors. Tourism Management. 36 541-551.

Ellen, T., \& Zhang, R. (2014). Measuring the Effect of Company Restaurant Servicescape on Patrons' Emotional States and Behavioral Intentions. Journal of Foodservice Business Research. 17(2) 85-102.

Fernandes, T., \& Neves, S. (2014). The role of servicescape as a driver of customer value in experience-centric service organizations: the Dragon Football Stadium case. Journal of Strategic Marketing. 22 (6) 548-560.

Grace, D., \& O'Cass, A. (2005). Service branding: Consumer verdicts on service brands. Journal of Retailing and Consumer Services. 12 (2) 125-139.

Harris, L. C., \& Ezeh, C. (2008). Servicescape and loyalty intentions: An empirical investigation. European Journal of Marketing. 42 (3-4) 390-422.

Harris, L. C., \& Goode, M. M. H. (2010). Online servicescapes, trust, and purchase intentions. Journal of Services Marketing. 24 (3) 230-243.

Hightower Jr, R., Brady, M. K., \& Baker, T. L. (2002). Investigating the role of the physical environment in hedonic service consumption: An exploratory study of sporting events. Journal of Business Research. 55 (9) 697-707.

Hightower, R. (2013). Investigating the green Leadership in Energy and Environmental Design (LEED) servicescape scale in Brazil. Construction Innovation: Information, Process, Management. 13 (3) 242-265.

Hightower, R. (2013). Investigating the green Leadership in Energy and Environmental Design (LEED) servicescape scale in Brazil. Construction Innovation. 13 (3) 242-265.

Hooper, D., Coughlan, J., \& Mullen, M. R. (2013). The servicescape as an antecedent to service quality and behavioral intentions. Journal of Services Marketing. 27 (4) 271-280.

IBGE. (2015) Contas Nacionais Trimestrais - Indicadores de Volume e valores Correntes. Sistema de contas nacionais Brasil, Julho / Setembro.

Ismail, A. R. (2011). Experience marketing: An empirical investigation. Journal of Relationship Marketing. 10 (3) 167-201.

Kim, W. G., \& Moon, Y. J. (2009) Customers' cognitive, emotional, and actionable response to the servicescape: A test of the moderating effect of the restaurant type. International Journal of Hospitality Management. 28 (1) 144-156.

Koo, G. Y., Andrew, D. P. S., \& Kim, S. (2008). Mediated relationships between the constituents of service quality and behavioural intentions: A study of women's college basketball fans. International Journal of Sport Management and Marketing. 4 (4) 390-411. Kotler, P. (1973). Atmospherics as a marketing tool. Journal of retailing. 49 (4) 48-64. Lam, L. W., Chan K. W., Fong, D., \& Lo, F (2011). Does the look matter? The impact of casino servicescape on gaming customer satisfaction, intention to revisit, and desire to stay. International Journal of Hospitality Management. 30 (3) 558-567.
LeBlanc, G., \& Nguyen, N. (1996). An examination of the factors that signal hotel image to travellers. Journal of Vacation Marketing. 3 (1) 32-42.

Lee, C. J., Wang, Y. C., \& Cai, D. C. (2015). Physical factors to evaluate the servicescape of theme restaurants. Journal of Asian Architecture

Lee, S. Y., \& Kim, J. H. (2014). Effects of Servicescape on Perceived Service Quality, Satisfaction and Behavioral Outcomes in Public Service Facilities. Journal of Asian Architecture and Building Engineering. 13 (1) 125-131.

Lim, W. M. (2014). The Antecedents and Consequences of Customer Hedonism in Hospitality Services. Journal of Hospitality Marketing and Management. 23 (6) 626-651.

Lin, I. Y. (2004). Evaluating a servicescape: the effect of cognition and emotion. International Journal of Hospitality Management. 23 (2) 163-178.

Lund, D. J., \& Marinova, D. (2014). Managing revenue across retail channels: The interplay of service performance and direct marketing. Journal of Marketing. 78 (5) 99-118.

Mari, M., \& Poggesi, S. (2013). Servicescape cues and customer behavior: a systematic literature review and research agenda. The Service Industries Journal. 33(2) 171-199. Martineau, P. (1958). The personality of the retail store. Harvard Business Review. 36, 47-55.

Miles, P., Miles, G., \& Cannon, A. (2012). Linking servicescape to customer satisfaction: Exploring the role of competitive strategy. International Journal of Operations and Production Management. 32 (7) 772-795.

Minkiewicz, J., Evans, J., Bridson, K., \& Mavondo, F. (2011). Corporate image in the leisure services sector. Journal of Services Marketing. 25 (3) 190-201.

Morrison, A. M. (2012) Marketing de Hospitalidade e Turismo. São Paulo: Cengage Learning.

Naqshbandi Muzamil, M., \& Munir, R. S. (2011). Atmospheric elements and personality: Impact on hotel lobby impressions. World Applied Sciences Journal. 15 (6) 785-792.

Newman, A. J. (2007). Uncovering dimensionality in the servicescape: Towards legibility. Service Industries Journal. 27(1) 15-28

Nguyen, D. T., DeWitt, T., \& Russell-Bennett, R. (2012). Service convenience and social servicescape: Retail vs hedonic setting. Journal of Services Marketing. 26 (4) 265-277. Nguyen, N. (2006). The collective impact of service workers and servicescape on the corporate image formation. International Journal of Hospitality Management. 25 (2) 227-244.

O'Cass, A., \& Grace, D. (2008). Understanding the role of retail store service in light of self-image - Store image congruence. Psychology \& Marketing. 25 (6) 521-537.

Pantouvakis, A. (2010). The relative importance of service features in explaining customer satisfaction A comparison of measurement models. Managing Service Quality. 20 (4) 366-387.

Pantouvakis, A. (2012). Internal marketing and the moderating role of employees: An exploratory study. Total Quality Management and Business Excellence. 23 (2) 177-195.

Parasuraman, A., Zeithaml, V. A., \& Berry, L. L. (1985). A conceptual model of service quality and its implications for future research. the Journal of Marketing. 49 (4) 41-50.

Reimer, A., \& Kuehn, R. (2005). The impact of servicescape on quality perception. European Journal of Marketing. 39. (7-8) 785-808.

Reynolds, K. L., \& Harris, L. C. (2009). Dysfunctional Customer Behavior Severity: An Empirical Examination. Journal of Retailing. 85 (3) 321-335.

Riviezzo, A., de Nisco, A., \& Napolitano, M. R. (2009). Importance-performance analysis as a tool in evaluating town centre management effectiveness. Internationa Journal of Retail and Distribution Management. 37 (9) 748-764.

Rosenbaum, M. S., \& Massiah, C. (2011). An expanded servicescape perspective. Journal of Service Management. 22 (4) 471-490.

Rosenbaum, M. S., \& Montoya, D. Y. (2007). Am I welcome here? Exploring how ethnic consumers assess their place identity. Journal of Business Research. 60 (3) 206-214.

Roy, S. K., Lassar, W. M., \& Butaney, G. T. (2014). The mediating impact of stickiness and loyalty on word-of-mouth promotion of retail websites: A consumer perspective. European Journal of Marketing. 48 (9-10) 1828-1849.

Sci Verse. (2015). Scopus FAQs. Recuperado em 15 de abril de 2015, de: http//www.info.scopus.com.

Solomon, M. R., Marshall, G. W., Stuart, E. W. (2015) Marketing: Real People, Real Choices (8 ed). Pearson Education.

Tuzovic, S. (2008). Investigating the concept of potential quality: An exploratory study in the real estate industry. Managing Service Quality. 18 (3) 255-271.

Wang, C. H., Hsu, L. C., \& Fang, S. R. (2009). Constructing a relationship-based brand equity model. Service Business. 3 (3) 275-292.

Zeithaml, V. A., Bitner, M. J., \& Gremler, D. D. (2014). Marketing de Serviços: A Empresa com Foco no Cliente. (6 ed) McGraw Hill Brasil.

Recebido: 02.02.2016

Reenviado: 05.07.2016

Aceite: 07.07.2016 